\title{
Estimating the Size of the Point Spread Function Using Fuzzy Logic for Blind Restoration of Satellite Image
}

\author{
https://doi.org/10.3991/ijes.v5i4.7849 \\ Fouad Aouinti $\left.{ }^{\varpi}\right)$, M'barek Nasri, Mimoun Moussaoui \\ Mohammed I University, Oujda, Morocco \\ aouinti.fouad@gmail.com
}

\begin{abstract}
Despite the considerable progress in the field of imaging, the acquired image can undergo certain degradations which are mainly summarized in blur and noise. The objective of the restoration is to estimate from the observed image an image as close as possible to the original image. The iterative blind deconvolution (IBD) can be used effectively when no information about the distortion is known. This algorithm starts with a random initial estimate of the point spread function (PSF) whose its size affects strongly the restoration process of the degraded image. In this paper, we have implemented a fuzzy inference system (FIS) to determine the size of the PSF through the examination of the blurred satellite image edges and the measurement of the blur width in pixels around an obviously sharp object. The obtained results are encouraging, which confirms the good performance of the proposed approach.
\end{abstract}

Keywords-Satellite image, PSF, edge detection, image restoration, IBD algorithm, fuzzy logic

\section{Introduction}

The research in the field of satellite imagery is considerably evolved in recent decades. However, the satellite image remains now limited in quality because of degradation effects which are due, on the one hand, to the intrinsic properties of instruments, and on the other hand, to the acquisition conditions [1]. The light coming from the original scene first passes through the atmosphere where it is subjected to the effects of turbulence, then the optical system where diffraction, defocusing and aberration act. The image thus arriving at the sensor is blurred. The latter is also contaminated by noise from various sources [2].

In order to overcome these distortions and produce a better image, two ways of improvement are possible. The first way is material, it consists of developing the new imaging devices, while the second way is the numerical methods, it seeks to reverse the process of image formation that is modeled by mathematical formulas. The digital restoration step is therefore indispensable to correct as much as possible the errors caused by the degradations [3]. Indeed, the image restoration is a problem that has occupied different scientific communities, its object is the reduction or even the elimination of the deterioration present in the acquired images. It is sufficient to know the 
parameters of blur and noise which are sometimes accessible, but in practice they are generally unknown. Unlike the classical filtering techniques, the blind deconvolution does not require any prior knowledge of the PSF, hence, it can be lot more useful in practical situations. The IBD is a very common algorithm for its low computational complexity, it restores the image and the PSF simultaneously based on a random initial estimate of the PSF whose its size is more important than the values it contains. In a real application, PSFs of different sizes are experimented, until the achievement of a satisfactory result [4].

In this work, we rely on the edge detection and the fuzzy logic, and we assume that the blur linked to the image is spatially invariant. The developed approach revolves around the blind restoration of degraded satellite image through the estimation of the appropriate PSF size using fuzzy logic. In order to ensure this, we design a FIS for deciding the PSF size to give to the IBD in terms of the maximum difference representing the sharp change of the luminous intensity of the unique edges image and of the maximum intensity of the same unique edges image.

The paper is organized as follows: in Section 2, we indicate some related works. In Section 3, we introduce the degradation process of satellite image and the IBD algorithm. Section 4 presents in detail our proposed approach based on fuzzy logic, which consists of determining the PSF size for blind image restoration. In Section 5, we assess the effectiveness of our idea by some experimental results. Finally, in Section 6 , we give a conclusion.

\section{Related works}

In the literature, several methods and approaches of image restoration have been proposed. The Wiener deconvolution processes the images degraded by both spatially invariant blur and additive noise, it attenuates the sensitivity of noise through the minimizing the mean square error between the original image and the estimated image [3]. In [5], a new technique for the satellite image deconvolution based on nonlocal means is proposed. An overview of the blind image deconvolution through the problem formulation and existing approaches has been introduced in [4]. The general technique adopted by IBD algorithm consists of deconvolving the degraded image using the iterative process, returning both the deblurred image and a restored PSF which are constrained in some manner. The restored PSF is a positive array that has the same size as initial PSF [6]. The paper [7] proposes a new regularized RichardsonLucy algorithm for remote sensing image deconvolution, a piecewise local regularization term is introduced to make the standard Richardson-Lucy algorithm capable of suppressing noise and ringing artifacts, and the proposed method is combined with residual deconvolution to strengthen the image edges. A new approach has been developed, in which a rough deconvolution is followed by noise filtering in the wavelet transform domain [8]. The paper [9] presents a new practical deblurring method, small-support-regularized (SSR) deconvolution, for low quality remotely sensed imagery. The aim of [3] is to optimize the compression/restoration chain in the context of satellite imaging through the proposition of a reliable technique to address the 
optimal decoding-deconvolution-denoising problem. The paper [10] develops an iterative algorithm based on convex optimization techniques and a wavelet frame regularization for restoration of satellite image degraded by spatially variant PSF.

The fuzzy sets theory is a generalization of the classical sets theory, which means that the latter is only a special case of the theory of fuzzy sets [11]. The fuzzy logic allows to take into account inaccuracies and uncertainties, one of his interests for formalizing human reasoning is that the rules are spelled out in natural language [12].

\section{Blind image restoration}

\subsection{Satellite image degradation}

The modeling of degradations suffered by the observed image is an essential step in order that the restoration takes place in good conditions. Consequently, the degradation process of a satellite image subjected to spatially invariant blur and noise, commonly adopted in the literature, is modeled by [13].

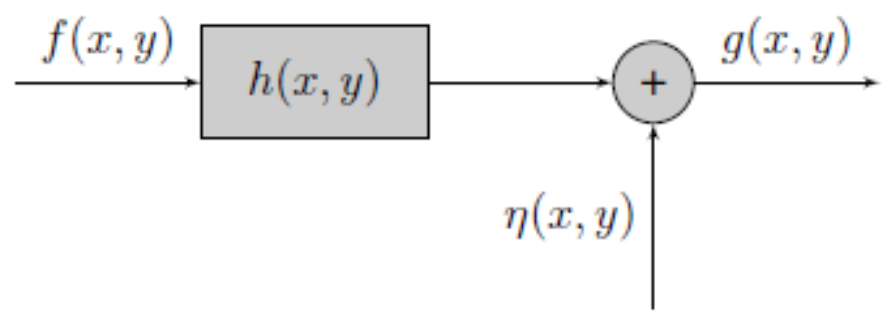

Fig. 1. Image degradation model

In the spatial domain, the modeling shown in figure 1 is denoted as follows:

$$
g(x, y)=f(x, y) * h(x, y)+\eta(x, y)
$$

where $g$ represents the degraded image, $f$ is the original image, $*$ denotes the convolution operator, $h$ is the degradation function which is also known as PSF, and $\eta$ is the Gaussian additive noise.

Since the representation of the digital image is discrete, the equation (1) can be expressed as:

$g(x, y)=\sum_{m=-k}^{k} \sum_{n=-k}^{k} f(x-m, y-n) \times h(m, n)+\eta(x, y)$

where the size of mask $h$ changes according to the parameters of blur.

In the frequency domain, the convolution product shown in equation (1) becomes a simple product of Hadamard (element by element):

$$
G(u, v)=F(u, v) H(u, v)+N(u, v)
$$


where $G, \mathrm{~F}, H$ and $N$ represent the two-dimensional Fourier transforms of $g, f, h$ and $\eta$ respectively.

\subsection{Iterative blind deconvolution}

The deconvolution consists of finding an estimate $\hat{f}$ of the original image $f$ from the observation $g$ as given by equation (1), it is therefore an inverse problem. Because of the noisy data, this problem is part of the ill-posed inverse problems class [14]. According to Hadamard, the problem of type $g=h(f)$ is well-posed if:

1. a solution exists;

2. the solution is unique;

3. the solution depends continuously on the data.

Thus, the solution may not exist, it is not necessarily unique and it may be unstable in other words a small error on the observation can lead to a large variation on the reconstructed solution.

In order to reconstruct the image $\hat{f}$, it is essential to determine the degradation parameters of blur and noise. These parameters are commonly unknown, then they must be estimated from the degraded image as shown in Figure 2.

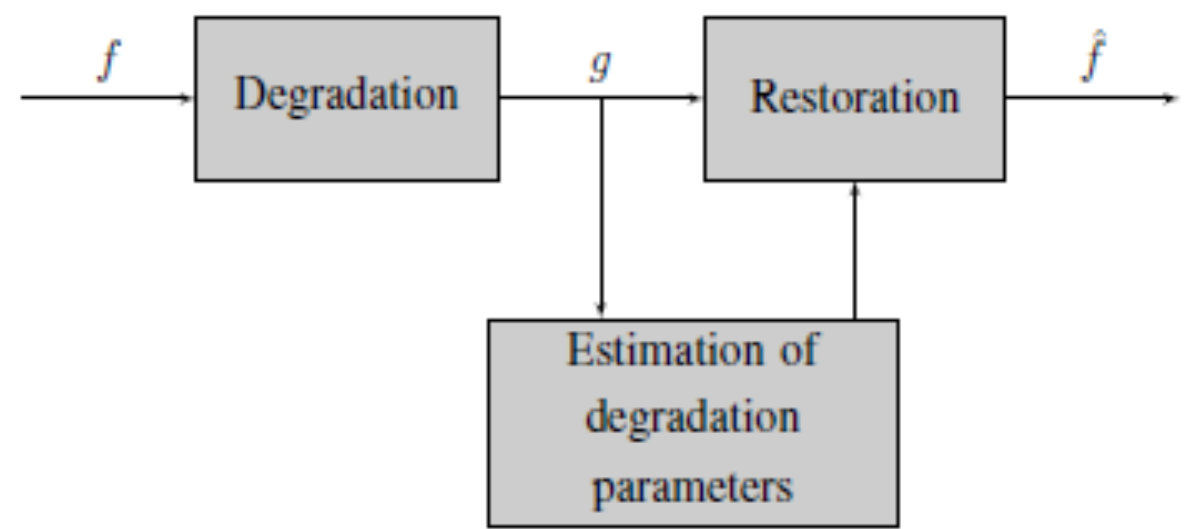

Fig. 2. Image restoration principle

In the literature, there are two main approaches for blind image restoration [4]:

1. identifying the PSF separately in order to use it later with one of the known classical image restoration methods;

2. estimating the PSF and the restored image simultaneously, which leads to the development of more complex algorithms.

The first approach uses some simple algorithms and methods to estimate the PSF, and thus, the result of restored image is not perfect. Therefore, in this paper, we are interested in the second approach. Many methods have been proposed in previous 
researches, among which the IBD method that is the most popular algorithm in the second class of approaches.

The IBD algorithm begins with an initial estimate $\hat{h}_{0}(x, y)$, and uses it with a traditional deconvolution to get the initial image estimate $\hat{f}_{0}(x, y)$. The following sequences define the algorithm at iteration $i$ :

Algorithm 1 IBD algorithm

Step 1: $\quad$ Find $\hat{F}_{i}(u, v)$ the discrete Fourier transform of $\hat{f}_{i}(x, y)$.

Step 2: Impose blur constraints in the Fourier domain (where (.)* denotes the complex conjugate):

$$
\widetilde{H}_{i}(u, v)=\frac{G(u, v) \hat{F}_{i}^{*}(u, v)}{\left|\hat{F}_{i}(u, v)\right|^{2}+\alpha /\left|\widehat{H}_{i-1}(u, v)\right|^{2}}
$$

Step 3: $\quad$ Find $\tilde{h}_{i}(x, y)$ the inverse discrete Fourier transform of $\widetilde{H}_{i}(u, v)$.

Step 4: Impose spatial domain positivity and finite support constraints on $\tilde{h}_{i}(x, y)$ to give $\hat{h}_{i}(x, y)$.

Step 5: $\quad$ Find $\widehat{H}_{i}(u, v)$ the discrete Fourier transform of $\widehat{h}_{i}(x, y)$.

Step 6: Impose image constraints in the Fourier domain:

$$
\tilde{F}_{i}(u, v)=\frac{G(u, v) \widehat{H}_{i}^{*}(u, v)}{\left|\widehat{H}_{i}(u, v)\right|^{2}+\alpha /\left|\widehat{F}_{i}(u, v)\right|^{2}}
$$

Step 7: $\quad$ Find $\tilde{f}_{i}(x, y)$ the inverse discrete Fourier transform of $\widetilde{F}_{i}(u, v)$.

Step 8: Impose spatial domain positivity and finite support constraints on $\tilde{f}_{i}(x, y)$ to give $\hat{f}_{i+1}(x, y)$.

Step 9: Next iteration: set $i=i+1$; go to step 1 .

The real constant $\alpha$ represents the energy of the additive noise, and it must be chosen carefully in order to obtain a reliable restoration. This algorithm is highly sensitive to the size of the initial guess of the PSF which has a direct impact on the expected result. The figure 3 illustrates the importance of knowing the size of the true PSF: 


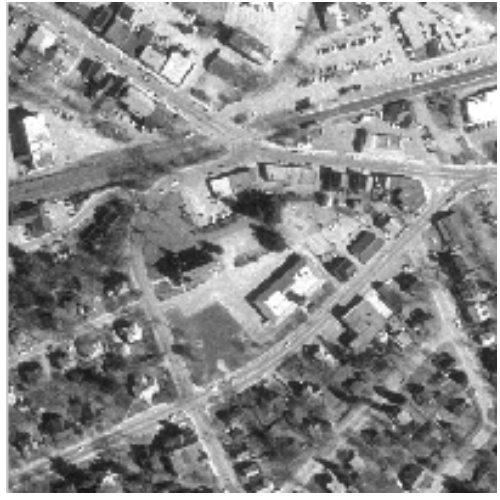

(a)

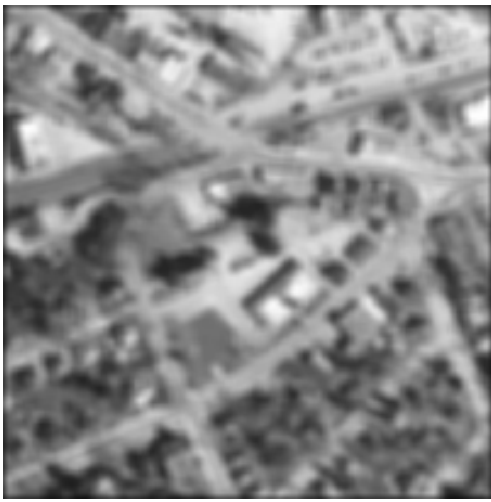

(c)

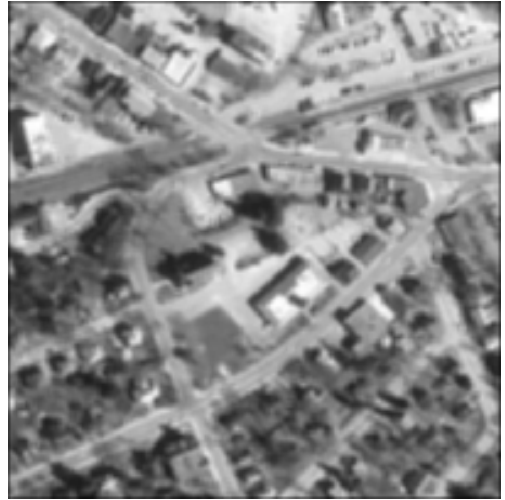

(b)

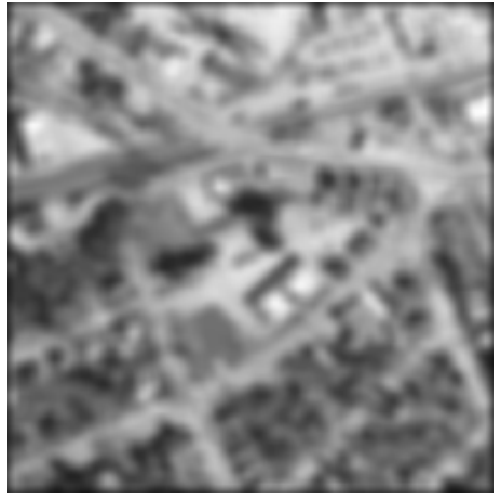

(d)

Fig. 3. Influence of the PSF size on the image. (a) Original image, (b) Degraded image with PSF size $5 \times 5$, (c) Degraded image with PSF size $9 \times 9$, (d) Degraded image with PSF size $13 \times 13$

\section{$4 \quad$ Proposed approach}

The edges constitute an essential information for estimating the strength of PSF effect on the degraded image. The Sobel filters are the processed filter set:

$$
h=\left(\begin{array}{ccc}
1 & 2 & 1 \\
0 & 0 & 0 \\
-1 & -2 & -1
\end{array}\right) \quad v=\left(\begin{array}{ccc}
-1 & 0 & 1 \\
-2 & 0 & 2 \\
-1 & 0 & 1
\end{array}\right)
$$

The unique edge image $E$ that does not take orientation into account is calculated as follows:

$$
E=\sqrt{E_{h}^{2}+E_{v}^{2}}
$$


where $E_{h}$ and $E_{v}$ are two images containing the horizontal and vertical edges respectively.

The fuzzy logic is based on the mathematical theory of fuzzy sets, it is an extension of classical logic that allows the modeling of data imperfections and approximates to a certain degree of human reasoning flexibility [11]. The main goal of the proposed Mamdani FIS is to decide the appropriate PSF size $S_{P S F}$ according to the maximum difference $D_{E}$ representing the sharp change of the luminous intensity of the unique edges image and to the maximum intensity $I_{E}$ of the same unique edges image. It is necessary to specify membership function for each fuzzy subset of each of our three linguistic variables:

1. input 1: $D_{E}$; subsets: low, normal, high;

2. input 2: $I_{E}$; subsets: black, white;

3. output: $S_{P S F}$; subsets: small, medium, large.

The form of each membership function is chosen by carrying out statistical studies in the figures 4, 5 and $6[15]$ :

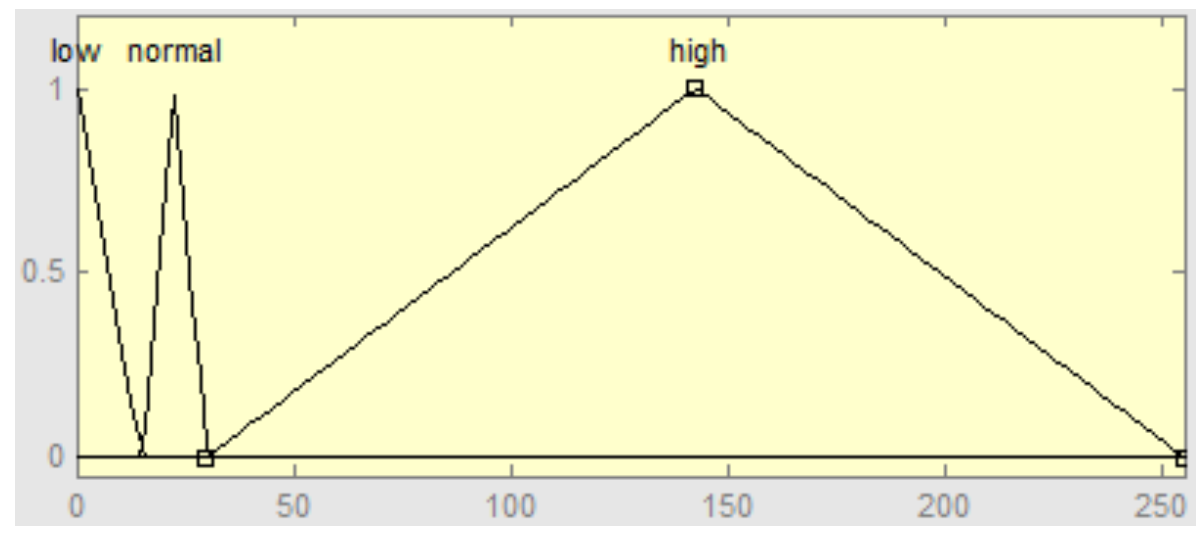

Fig. 4. Input variable diff

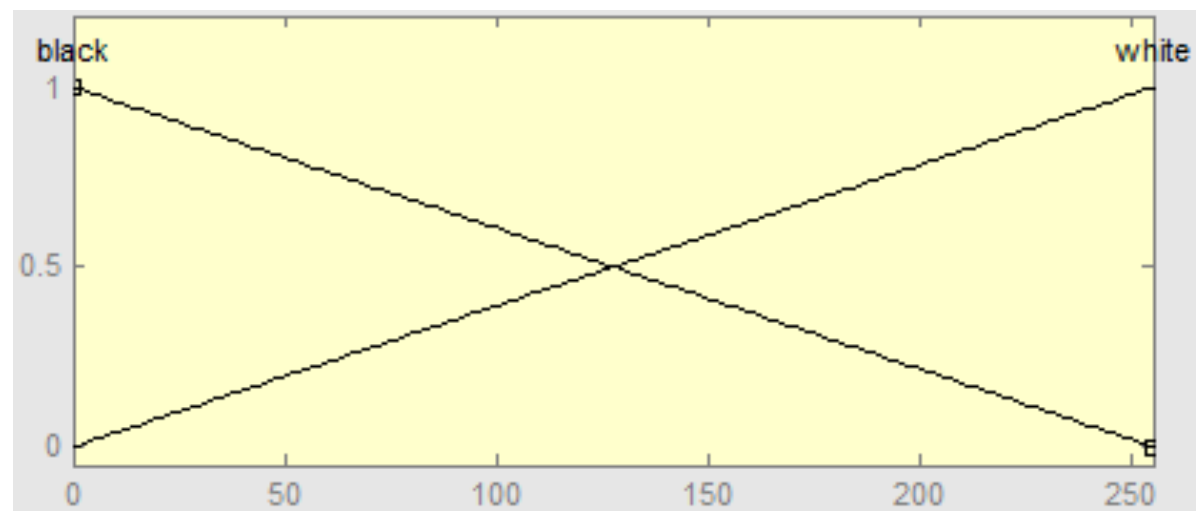

Fig. 5. Input variable intens 


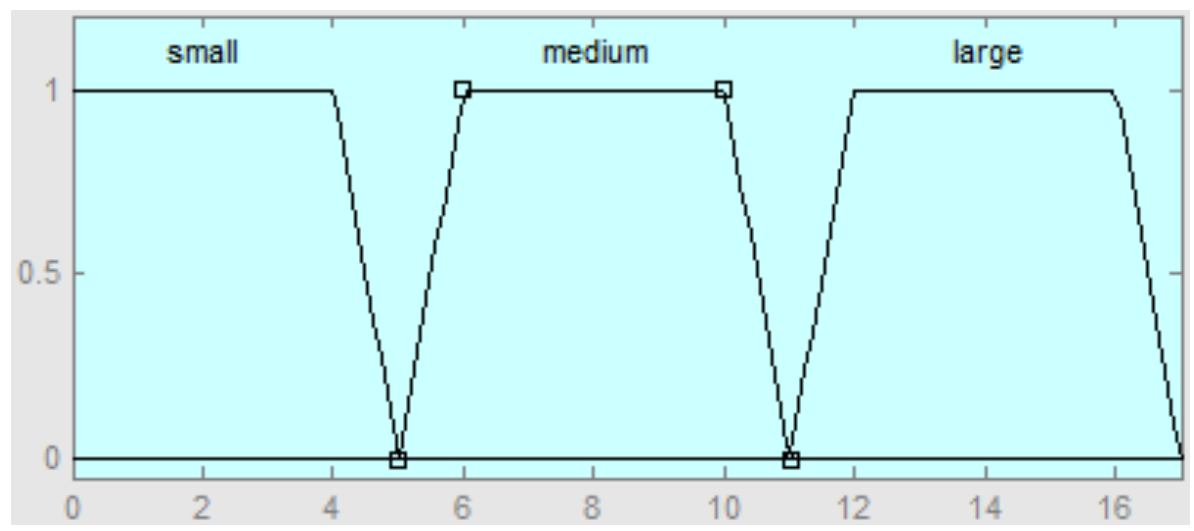

Fig. 6. Output variable size

In order to manipulate the fuzzy sets easily, we select the operators of Zadeh MIN/MAX for And/Or methods to adapt them to the membership functions:

$$
\begin{aligned}
& \mu_{D_{E} \cap I_{E}}(x)=\min \left(\mu_{D_{E}}(x), \mu_{I_{E}}(x)\right) \\
& \mu_{D_{E} \cup I_{E}}(x)=\max \left(\mu_{D_{E}}(x), \mu_{I_{E}}(x)\right)
\end{aligned}
$$

The fuzzy reasoning is based on fuzzy rules that are expressed in natural language using the linguistic variables of which we have defined previously [16]:

1. If (diff is high) and (intens is white) then (size is small);

2. If (diff is normal) then (size is medium);

3. If (diff is low) and (intens is black) then (size is large).

\section{Experimental results and evaluations}

The test satellite image used in this work is a grayscale image of the "Image Processing Toolbox" proposed by the MATLAB software [17], while the fuzzy logic is implemented through the "Fuzzy Logic Toolbox" also developped by MATLAB [18]. When the causes of blur are various and complicated, a Gaussian degradation model is used to approximate the composite effect.

A study is performed on an image subjected to the degradation of both the Gaussian blur (mask of $7 \times 7$, standard deviation $\sigma_{h}=5$ ), and the Gaussian additive noise (mean $\mu_{\eta}=0$, variance $\sigma_{\eta}^{2}=0.00001$ ). The estimation of the PSF size is presented in figure 7. 


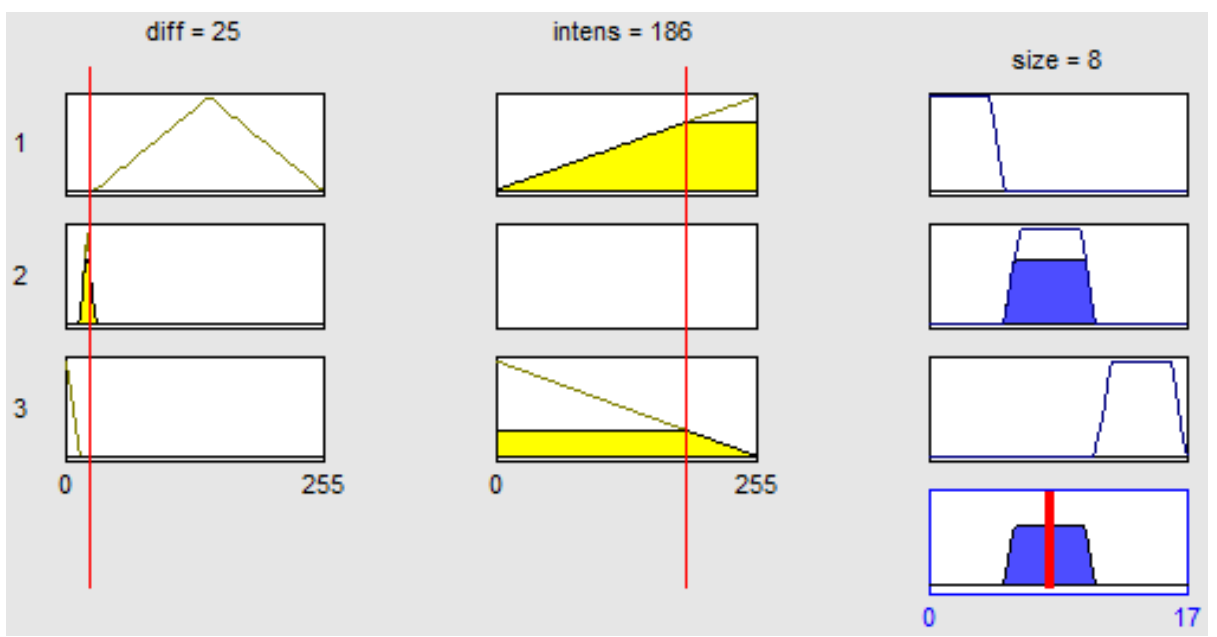

Fig. 7. Defuzzification with the centroid method

The image quality is evaluated by the Peak Signal-to-Noise Ratio (PSNR) quality measure:

$$
\operatorname{PSNR}(f, \hat{f})=10 \log _{10}\left(\frac{R^{2}}{\|\hat{f}-f\|_{2}^{2}}\right)(d B)
$$

where $R$ is the maximum value of the image pixels intensities. In our case, $R=255$.

The figure 8 shows the obtained result of the realized study.

The image quality improves which is illustrated in figure 8 , demonstrating that the blind restoration of the satellite image using proposed FIS gives a better result and remains effective when the image is degraded by both Gaussian blur and Gaussian additive noise.

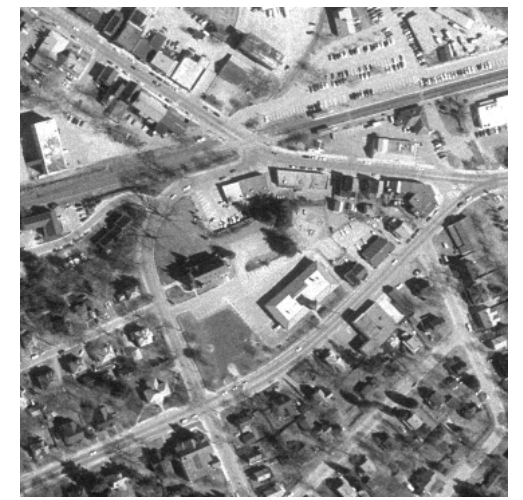

(a)

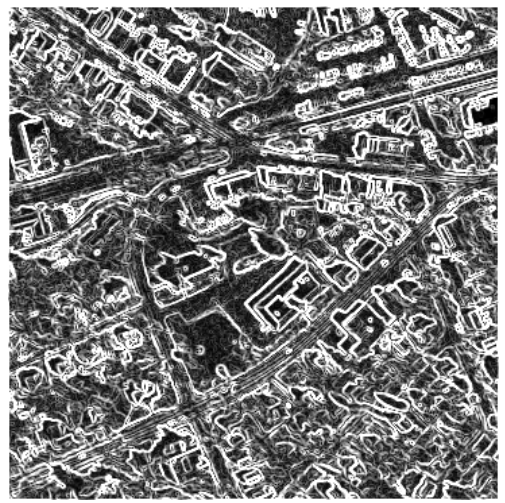

(b) 


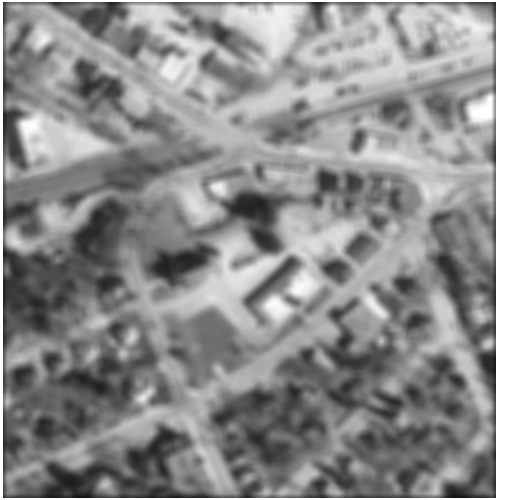

(c)

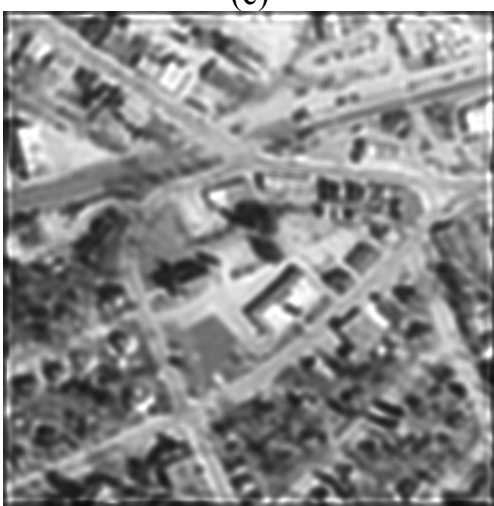

(e)

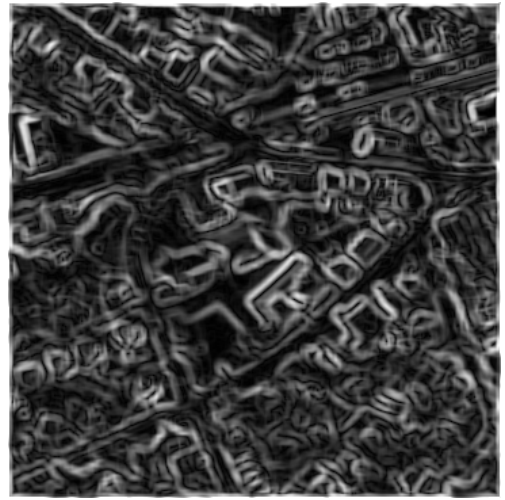

(d)

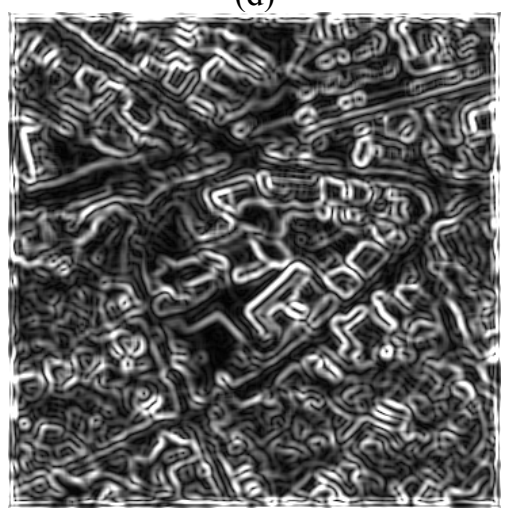

(f)

Fig. 8. Unique edges detection using the Sobel method. (a) Original image, (b) Edges of the original image, (c) Degraded image, (d) Edges of the degraded image, (e) Restored image $P S N R=17.28 \mathrm{~dB}$, (f) Edges of the restored image

\section{Conclusion}

We have proposed an improvement of IBD algorithm based on fuzzy logic in order to get rid of the difficulties related to the initialization of the PSF size parameter. To do this, we have defined the most suitable FIS. Indeed, the result of realized study affirms the efficiency of the proposed approach that is useful and valid as long as the satellite image to restore is weakly or moderately degraded. For the execution, the presented approach ensures a reasonable calculation time.

Despite the IBD algorithm is affected heavily by the size of the initial guess of PSF and less by the values it contains, the estimating of these values remains an important step, and thus, the knowledge of blur form using fuzzy logic for blind image restoration may be the subject of a study thereafter. 


\section{$7 \quad$ References}

[1] Jalobeanu A., Blanc-Féraud L., \& Zerubia J. (2004). An adaptive Gaussian model for satellite image deblurring. IEEE Transactions on Image Processing, 13(4):613-621. https://doi.org/10.1109/TIP.2003.819969

[2] Molina R., Nunez J., Cortijo F. J., \& Mateos J. (2001). Image Restoration in Astronomy: A Bayesian Perspective. IEEE Signal Processing Magazine, 18(2):11-29. https://doi.org/10.1109/79.916318

[3] Carlavan M. (2013). Optimization of the compression/restoration chain for satellite images. Ph.D. thesis, Université de Nice - Sophia Antipolis, France.

[4] Campisi P., \& Egiazarian K. (2007). Blind Image Deconvolution: Theory and Applications. CRC Press. https://doi.org/10.1201/9781420007299

[5] Zhao M., Zhang W., Wei W., Wang Z., \& Hou Q. (2010). Satellite image deconvolution based on nonlocal means. Applied Optics, 49(32):6286-6294. https://doi.org/10.1364/AO. 49.006286

[6] Molina R., Mateos J., \& Katsaggelos A. K. (2006). Blind deconvolution using a variational approach to parameter, image, and blur estimation. IEEE Transactions on Image Processing, 15(12):3715-3727. https://doi.org/10.1109/TIP.2006.881972

[7] Dong W., Feng H., Xu Z., \& Li Q. (2011). A piecewise local regularized Richardson-Lucy algorithm for remote sensing image deconvolution. Optics \& Laser Technology, 43(5):926-933. https://doi.org/10.1016/j.optlastec.2010.12.012

[8] Jalobeanu A., Blanc-Féraud L., \& Zerubia J. (2003). Satellite Image Deblurring Using Complex Wavelet Packets. International Journal of Computer Vision, 51(3):205-217. https://doi.org/10.1023/A:1021801918603

[9] Li Y., \& Clarke K. C. (2013). Image deblurring for satellite imagery using small-supportregularized deconvolution. ISPRS Journal of Photogrammetry and Remote Sensing, 85:148-155. https://doi.org/10.1016/j.isprsjprs.2013.08.002

[10] Hajlaoui N., Chaux C., Perrin G., Falzon F., \& Benyahia B. (2010). Satellite image restoration in the context of a spatially varying point spread function. JOSA A, 27(6):14731481. https://doi.org/10.1364/JOSAA.27.001473

[11] Zadeh L. A. (2008). Is there a need for fuzzy logic?. Information sciences, 178(13):27512779. https://doi.org/10.1016/j.ins.2008.02.012

[12] Zadeh L. A. (1973). Outline of a new approach to the analysis of complex systems and decision processes. IEEE Transactions on Systems, Man, and Cybernetics, 3(1):28-44. https://doi.org/10.1109/TSMC.1973.5408575

[13] Jalobeanu A., Blanc-Féraud L., and Zerubia J. (2002). Hyperparameter estimation for satellite image restoration using a MCMC maximum-likelihood method. Pattern Recognition, 35(2):341-352. https://doi.org/10.1016/S0031-3203(00)00178-3

[14] Burger M., \& Scherzer O. (2001). Regularization methods for blind deconvolution and blind source separation problems. Mathematics of Control, Signals and Systems, 14(4):358-383. https://doi.org/10.1007/s498-001-8041-y

[15] Mamdani E. H., \& Assilian S. (1975). An experiment in linguistic synthesis with a fuzzy logic controller. International Journal of Man-Machine Studies, 7(1):1-13. https://doi.org/10.1016/S0020-7373(75)80002-2

[16] Nachtegael M., van der Weken D., van de Ville D., \& Kerre E. E. (2003). Fuzzy Filters for Image Processing. Springer-Verlag Berlin Heidelberg. https://doi.org/10.1007/978-3-54036420-7

[17] MathWorks, MATLAB. https://www.mathworks.com/ 
Paper-Estimating the Size of the Point Spread Function Using Fuzzy Logic for Blind Restoration of...

[18] Sivanandam S. N., Sumathi S., \& Deepa S. N. (2007). Introduction to Fuzzy Logic using MATLAB. Springer Berlin Heidelberg. https://doi.org/10.1007/978-3-540-35781-0

\section{Authors}

Fouad Aouinti received an engineering diploma in computer science in 2013 from National School of Applied Sciences, Tetouan, Morocco. He is a PhD student who works on image processing in MATSI laboratory of the Superior School of Technology, Mohammed I University, Oujda, Morocco. His main field of research interest is image restoration based on genetic algorithm, fuzzy logic and neural networks.

M'barek Nasri is Engineer of the National School of the Mineral Industry, Rabat, Morocco. He is Doctor in Science (PhD degree, 2004) of the Mohammed I University, Oujda, Morocco. He obtained the Habilitation degree in 2006 from the same University. He teaches the computer science. His field of research interest is in image processing and computer vision and their applications to the medical imaging, quality control and recognition of the handwritten writing. He is permanent member of the MATSI laboratory.

Mimoun Moussaoui received his doctorate in Numerical Analysis in 1984 from University Paris XI (Orsay, France). He obtained his PhD in Nonlinear Analysis in 1991 from the Free University (Université Libre) of Brussels Belgium. He is currently professor at the Mohammed I University of Oujda (Morocco). He teaches mathematics for economics and scientists, numerical analysis and linear programming. He supervised several theses in applied mathematics and computing. He is director of mathematics, signal and image processing and computing research laboratory (MATSI).

Article submitted 17 October 2017. Published as resubmitted by the authors 20 November 2017. 